\title{
A Review of Language, Executive Function, and Intervention in Autism Spectrum Disorder
}

\author{
Laura Friedman, M.S. ${ }^{1}$ and Audra Sterling, Ph.D. ${ }^{1}$
}

\section{ABSTRACT}

Difficulties with both executive functions and language skills are common but variable in autism spectrum disorder (ASD). Executive functions and language skills are related to one another, such that vocabulary, syntax, and pragmatics are related to domains of working memory, shifting, and inhibition in ASD, although the directionality of these relationships remains unclear. Moreover, interventions that target pragmatic ability have been found to improve executive function skills, and conversely, executive function interventions are linked with improvements in social skills in children with ASD. We review the literature on executive functions, language skills, and their relationship in ASD; discuss factors that may be driving inconsistent findings; and explore clinical applications from the research thus far.

KEYWORDS: autism spectrum disorder, executive function, language, intervention

Learning Outcomes: As a result of this activity, the reader will be able to (1) explain the relationship between executive functions and language in ASD; (2) describe how targeted interventions may impact executive function or language performance in ASD; and (3) explain how various executive function task features and inclusion of different comparison groups may yield distinctive findings.

Autism spectrum disorder (ASD) is a neurodevelopmental disorder that is behaviorally diagnosed and is characterized by deficits in social communication and the presence of restricted and repetitive patterns of behavior. ${ }^{1}$ Recent estimates from the Centers for Disease Control and Prevention indicate that this disorder affects approximately 1 in 59 children. $^{2}$
${ }^{1}$ Department of Communication Sciences and Disorders, University of Wisconsin-Madison, Madison, Wisconsin.

Address for correspondence: Laura Friedman, M.S., Department of Communication Sciences and Disorders, University of Wisconsin-Madison, 1975 Willow Drive, Madison, WI 53706 (e-mail: Laura.friedman@wisc.edu).

Executive Function Skills in Children with Language Differences and Disorders; Guest Editor, Margarita

Kaushanskaya, Ph.D.
Semin Speech Lang 2019;40:291-304. Copyright (C) 2019 by Thieme Medical Publishers, Inc., 333 Seventh Avenue, New York, NY 10001, USA. Tel: +1(212) 584-4662. DOI: https://doi.org/10.1055/s-0039-1692964.

ISSN 0734-0478. 
Children, adolescents, and adults with ASD often exhibit deficits in various aspects of executive function, although numerous review articles and meta-analyses indicate variability in executive function performance in this population, depending on the tasks and comparison groups included in the study. ${ }^{3-7}$ Executive functions are defined as cognitive processes that underlie goal-directed behavior, and include skills such as shifting or cognitive flexibility, inhibition, and working memory, as well as higher-level functions such as planning..$^{8-10}$ Executive functions have a vast impact on daily life, and are closely related to the ability to communicate with others. ${ }^{3,11-13}$ Understanding the relationship between communicative skills and executive function skills is important because both domains have impacts on learning, scholastic achievement, and social development. ${ }^{14-16}$ Additionally, various theories have proposed that language mediates executive functions, while others have proposed that executive functioning underlies communication skills. ${ }^{11,17,18}$ This article reviews empirical studies that examined executive functions (e.g., shifting, inhibition, working memory, and planning) and language (e.g., semantics, syntax, and pragmatics) in ASD, and discusses clinical implications drawn from existing evidence.

\section{LANGUAGE OVERVIEW IN AUTISM SPECTRUM DISORDER}

Social communication impairments are a core characteristic and required to obtain a behavioral diagnosis of ASD. Pragmatic language is one component of social communication and includes a variety of skills. Children with ASD have challenges with topic maintenance, greetings and farewells, appropriate turn taking, and using conversational repair strategies. These deficits are associated with challenges with perspective-taking and theory of mind. ${ }^{19}$ Due to the pervasiveness of pragmatic language deficits, this area has been extensively studied in ASD. ${ }^{20-22}$ Children with ASD are impaired in their pragmatic skills compared with peer groups with typical development (TD), intellectual disability, or other disorders. ${ }^{23-25}$

While deficits in pragmatics and social communication are a hallmark feature of
ASD, structural language abilities, specifically semantics and syntax, vary and result in marked individual differences. Several studies indicate a discrepancy in expressive and receptive vocabulary scores, such that expressive vocabulary scores are higher than receptive vocabulary scores. ${ }^{26-28}$ Other studies have noted no difference, or reported higher receptive vocabulary scores than expressive vocabulary scores, and the discrepant findings appear to depend on the measures and ages of participants. ${ }^{28-30}$ In addition to deficits in standardized vocabulary scores, children with ASD present with unique deficits in specific subdomains of vocabulary, including the production and comprehension of personal pronouns, mental state terms, and prepositions. ${ }^{23,31,32}$ These subdomains of vocabulary may be particularly impacted because they require perspective-taking, which is often a challenge for individuals with ASD. For example, use and understanding of mental state terms, which refer to one's emotional or cognitive state, require theory of mind. ${ }^{33}$ Similarly, correct use of personal pronouns requires the speaker to know if the communication partner is aware of the referent. If not, a communication breakdown can occur.

Within the domain of syntax, children and adolescents with ASD are variable in their deficits. Several studies have suggested that children with ASD exhibit syntactic delays in comparison to peers with intellectual disability or TD. ${ }^{34-37}$ These syntactic abnormalities can include challenges with verb phrases, regular and irregular past tense, present tense, and regular third person singular verbs. ${ }^{35,36}$ However, like deficits in semantics, challenges in this domain of language are not universal among individuals with ASD; several studies have reported intact syntactic skills in individuals with ASD when compared with TD peers. ${ }^{38}$ In fact, several studies have proposed that there exists a subgroup of children with ASD who have grammatical impairments that mirror those of children with developmental language disorder (DLD), which is a disorder characterized by deficits in language without a known etiology. ${ }^{39,40}$ However, general patterns have indicated that while some children with ASD have challenges with certain aspects of syntax, this is not true for all children with ASD. 


\section{EXECUTIVE FUNCTION OVERVIEW IN AUTISIM SPECTRUM DISORDER}

Similar to structural language, many children and adolescents with ASD have difficulties with executive functions, although unlike pragmatic language, this is not a hallmark deficit of this population. Many studies have found that executive functions are generally impaired at the group level in children and adolescents with ASD, particularly in comparison to peers with $\mathrm{TD},{ }^{41-43}$ although this is not always the case. ${ }^{44}$ It is important to be mindful of individual differences, though, as some children with ASD do not exhibit deficits in executive function, indicating that these deficits are not a core impairment in this population. ${ }^{45}$ It is also particularly difficult to draw conclusions about children with ASD because studies vary in group matching criteria, as well as inclusionary and exclusionary criteria. However, some studies that have conflicting findings report similar ages for their participant groups, as well as similar matching criteria, comparison groups, and covariates. ${ }^{42-44}$ Two common comparison groups are children with attention-deficit/ hyperactivity disorder (ADHD) and children with DLD. Specifically, comparisons between ASD and ADHD are frequent because these two disorders share phenotypic overlap, and understanding their respective executive function skills can shed light on similarities and differences in these groups. On the other hand, comparisons between children with ASD and children with DLD inform the relationship between language skills and executive functions. These groups are commonly compared when examining language development in ASD.

Executive function domains, as noted earlier, include shifting, inhibition, and working memory (commonly referred to as updating). These domains, although related, are separable and unique constructs, as indicated by a factor analysis completed by Miyake and colleagues. ${ }^{9}$ We will define these domains and summarize the literature on executive function skills in children and adolescents with ASD. We delve into more detail for the domain of planning, which is regarded as a higher-level cognitive skill, given that it is inherently more complex than shifting, inhibition, and working memory. ${ }^{9}$ Notably, some methods used to measure executive functions are verbal or linguistic in nature, while others are nonverbal or do not have a linguistic component. We will identify the verbal or linguistic nature of the tasks, as this feature may impact the interpretation of the findings. After examining executive functions in ASD, we will discuss studies that have specifically linked shifting, inhibition, working memory, and planning to language abilities.

Shifting. Shifting is the ability to switch between mental states or tasks and is also commonly referred to in the literature as cognitive flexibility. There is some evidence that shifting is impaired in ASD in comparison to peer groups with $\mathrm{DLD}^{46}$ and $\mathrm{ADHD},{ }^{42,43}$ as well as TD peers. ${ }^{47}$ However, other studies have not found differences in shifting between children with ASD and children with TD, intellectual disability, or ADHD. ${ }^{44,48}$ Several studies have used the Wisconsin Card Sorting Task $\left(\mathrm{WCST}^{49}\right)$ to examine shifting. In this task, participants are expected to shift between sorting cards based on one of three dimensions, including color, number, and shape. Although the WCST does not require a verbal output, linguistic skills can help the participant to verbally encode and rehearse the shapes or colors of the targets for sorting. A perseverative error occurs if an individual does not shift to the new sorting expectation and responds based on the previous sorting expectation. Children with ASD tend to make more of these perseverative errors during this task in comparison to peer groups with TD, ADHD, and DLD. ${ }^{46,50}$ For example, Liss and colleagues $^{46}$ compared shifting abilities between school-age children with high-functioning ASD and children with DLD matched on IQ, age, and socioeconomic status. Notably, the children with DLD had an average verbal IQ score that was 10 points higher than the children with ASD. Shifting was measured using the WCST. They found that the children with ASD made more perseverative errors on the WCST, but the difference in perseverative errors was no longer significant when accounting for verbal IQ. The authors ultimately proposed that there were no unique shifting deficits in ASD, and that verbal IQplayed a key role in shifting performance. 
A 2014 meta-analysis ${ }^{5}$ of shifting focused on reported data from a large sample of individuals with ASD and TD. Across a range of performance measures, the authors found that participants with ASD and TD were not different on shifting performance overall, although they did find a difference between groups on the Shift subscale of the Behavior Rating Inventory of Executive Function (BRIEF ${ }^{51,52}$ ). The authors of this meta-analysis advise that performance measures of cognitive flexibility may lack ecological validity, as these measures were not as sensitive as the BRIEF in distinguishing between clinical and control groups. Overall, they suggest that their results are not surprising given that cognitive inflexibility, or difficulty with shifting, is not a core characteristic of ASD. However, the authors reported that the majority of the studies included in the metaanalysis had compared individuals with ASD to age-matched controls, but the authors did not consider group matching status in their inclusionary criteria. ${ }^{5}$ Therefore, results from this meta-analysis may not generalize to all individuals with ASD; comparison groups should be considered in future work.

Inhibition. Response inhibition has been a focus of several studies that compare participants with ASD to various peer groups, often participants with ADHD and TD. This aspect of executive function involves suppressing information that may interfere with the task goal at hand. Like results for shifting, findings related to response inhibition in children and adolescents with ASD are varied. Some studies have reported no differences between children with ASD and their peer groups with ADHD and $\mathrm{TD},{ }^{44,48,53}$ while others have reported marked impairments in this domain. ${ }^{42,43}$

Several studies have compared verbal response inhibition performance in children with ASD to that in children with TD. For example, Yang and colleagues ${ }^{44}$ examined this skill using the Stroop task ${ }^{54}$ in children with ASD (mean age $=8.1$ years) in comparison to children with TD (mean age $=8.0$ years) and children with ADHD (mean age $=8.2$ years). Children were all statistically similar on age, and nonverbal IQ scores were controlled in their analyses. The Stroop task requires participants to provide a verbal output while suppressing other verbal information. For example, in this task the participant may see the word BLUE printed in green ink, and they must name the color of the ink instead of the printed word. The authors found no significant differences between the three groups in performance on the Stroop task as a measure of inhibition. ${ }^{44}$ Corbett and colleagues $^{42}$ examined response inhibition in similar groups of children, including children with high functioning ASD (mean age $=9.44$ years), TD (mean age $=9.56$ years), and ADHD (mean age $=9.40$ years). They controlled for IQ in their analyses, and measured inhibition with various tasks, including one verbal task that was similar to the Stroop test. On the Stroop-like task, they found that children with ASD performed worse on measures of inhibition compared with the children with TD, but they performed similarly with the children with ADHD. There were no differences between the children with ADHD and the children with TD on this task. The authors also measured response inhibition with a task in which participants pressed a button when they saw or heard a number "1" but not a number "2." Thus, this task did not require a verbal output, although the input was linguistic in nature. Children with ASD performed worse than children with TD on both tasks, but they only performed worse than children with ADHD on the visual number presentations. ${ }^{42}$

One study by Johnston and colleagues ${ }^{55}$ examined verbal response inhibition in adults with ASD (mean age $=27.8$ years) compared with adults with ADHD (mean age $=27.3$ years), and TD adults (mean age $=27.3$ years). The groups were matched on verbal IQ (all participants had verbal IQ scores within the normal range), age, and gender. The authors used verbal tasks, including the Stroop task ${ }^{54}$ and the Hayling Sentence Completion test, ${ }^{56}$ which requires participants to complete a sentence using either a relevant, connected word, or an irrelevant, unconnected word. Participants with ASD had typical response inhibition but slow response latencies. The authors suggested that this was likely due to deficits in response initiation. That is, adults with ASD were slower and more accurate, while adults with ADHD were more impulsive in the manner in which they completed the task. ${ }^{55}$ 
Working memory. Updating of working memory is the ability to revise recalled information as task goals update or change. ${ }^{14}$ Similar to the domains of shifting and inhibition, results from working memory tasks are variable: some studies have reported that children with ASD are impaired when compared with peer groups such as children with TD and children with $\mathrm{ADHD},{ }^{42,43}$ while others have reported no differences in working memory performance between individuals with ASD and peer groups with TD, ADHD, and intellectual disability. ${ }^{43,44,48}$

Roelofs and colleagues ${ }^{48}$ did not find differences in working memory among adolescents and young adults with ASD and intellectual disability, compared with age- and IQ-matched individuals without ASD. Updating of working memory was measured using a verbal fluency task and a verbal backward digit span test. Kado and colleagues $^{43}$ reported similar working memory performance between children with ASD and children with ADHD, but both groups had impaired working memory compared with chronological age- and IQ-matched school-age children with TD. In this study, the authors used a modified Japanese version of the $\mathrm{WCST}^{49}$ which included fewer cards. ${ }^{57}$ The test was purported to measure aspects of inhibition, shifting, and working memory. They presented several scores for this task, including the number of sorted sets of six consecutive cards. The authors found that children with ASD and ADHD sorted fewer sets and had a higher number of total errors than the children with TD, indicative of inadequate working memory. ${ }^{43}$

To tease out verbal demands of executive function tasks, some studies deliberately separate working memory into two domains: spatial or visual working memory and verbal working memory. For example, Hill and colleagues ${ }^{58}$ assessed working memory in 5- to 8-year-old children with ASD and children with DLD. The children with ASD were divided into two groups: children who had co-occurring language impairment and children who had typical or normal language abilities. They found that children with ASD and language impairment performed worse on verbal working memory tasks than the children with ASD and normal language. The children with ASD and language impairment and children with DLD performed similarly on most verbal working memory tasks except for a nonword repetition task, in which the children with DLD produced more errors. However, there were no group differences on the visual working memory tasks, indicating that the increased demands associated with a verbal working memory task may impact performance and differentiate ASD groups with and without language impairment. ${ }^{58}$

Planning. Planning is considered to be a higher-level cognitive process, and requires sequences of planned actions to be monitored and updated when given additional knowledge or when a preceding action occurs. ${ }^{6}$ Generally, children with ASD demonstrate marked deficits in planning compared with TD peers, as well as peer groups with ADHD and Tourette's syndrome, ${ }^{47,50,59}$ although children with ASD may have similar planning skills to children with a language disorder. ${ }^{46}$

Kimhi et al noted differences in planning ability between preschool children with ASD and chronological- and mental age-matched children with TD. ${ }^{47}$ Older children with ASD may also exhibit deficits in planning, as a longitudinal study examining executive function in adolescents with ASD relative to adolescents with learning disability found that adolescents with ASD have deficits in planning compared with the children with TD; notably, these deficits did not improve over a 3 -year time period. ${ }^{59}$ In contrast to these findings, Liss and colleagues did not find differences between boys with ASD and boys with DLD on the Mazes subtest from the Wechsler Intelligence Scale for ChildrenRevised, which is a task that measures aspects of planning, shifting, and inhibition. ${ }^{46}$ Also, Corbett and colleagues ${ }^{42}$ did not find significant differences in planning between children with high functioning ASD, children with ADHD, and children with TD when they used the Cambridge Neuropsychological Test Automated Battery (CANTAB) Stockings of Cambridge task, which is a spatial planning task.

Generally, it appears that some individuals with ASD exhibit deficits in areas of executive functions, but findings seem to be dependent on several factors. First, outcomes may be dependent on the ages of the participants. For example, 
studies that include adolescents or adults generally find that participants with ASD do not perform differently than TD peers or peers with ADHD or intellectual disability, ${ }^{44,48,53}$ whereas studies that only include school-age or younger participants are more likely to reveal differences. ${ }^{42,46}$ Second, verbal and nonverbal tasks can yield differences, such that tasks which require verbal responses generally differentiate groups, while tasks that are more visually based do not. ${ }^{58}$ This pattern of findings suggests that executive function skills by themselves may not impact in ASD, but group differences may emerge when executive function tasks include verbal or linguistic demands. While language deficits in the domains of vocabulary and syntax are not hallmark features of ASD, some children do experience delays in these domains. ${ }^{39}$ Therefore, it is important to include language-matched groups of children if selecting executive function tasks that require verbal output. For example, Hill and colleagues reported that groups of children with ASD and DLD matched on language ability performed similarly on verbal working memory tasks, ${ }^{58}$ highlighting the importance of language matching.

Many studies, however, do not include language-matched groups of children. Rather, groups are often matched on chronological age, mental age, or IQ. ${ }^{43,46,48,53}$ Selection of comparison groups and matching criteria are important to consider. For instance, comparing children with ASD to children with DLD may yield different results than comparing children with ASD to children with ADHD or other intellectual and developmental disabilities. A comparison of age- and IQ-matched children with ASD and DLD suggests similarities in inhibition, and shifting, and planning. ${ }^{46}$ Age-matched children with ASD and children with DLD do not differ in performance on visual working memory tasks, and children with ASD + language impairment and children with DLD perform similarly on verbal working memory tasks. ${ }^{58}$ In comparison to age- and IQ-matched children with ADHD, children with ASD either perform worse $e^{42,43}$ or similarly ${ }^{44}$ on shifting, inhibition, and working memory measures. Additionally, comparisons of individuals with ASD + intellectual disability and individuals with intellectual disability without ASD do not yield differences in shifting, inhibition, or working memory. ${ }^{48}$ Ultimately, these findings suggest that, when controlling for linguistic abilities and task demands, individuals with ASD are not likely uniquely experiencing executive dysfunction. Furthermore, language skills, along with comorbid diagnoses, such as ADHD, must be considered when assessing and targeting executive functions in children with this heterogeneous disorder.

To elucidate the interplay between executive functions and language, several studies have explored the relationship between these two domains in children and adolescents with ASD. We will review studies that have examined executive functions in relation to vocabulary, syntax, and/or pragmatic abilities.

\section{THE RELATIONSHIP BETWEEN EXECUTIVE FUNCTION AND LANGUAGE IN ASD}

Studies examining executive functions and language in children with TD have found correlations between these two domains. One particularly relevant model for conceptualizing the relationship between executive functions and language is the Hierarchical Competing Systems Model (HCSM). ${ }^{18}$ This model suggests that there is a habit system which is influenced by previous experience, and a representational system which is influenced by conscious reflection on behavior. These two systems are hierarchically arranged, and they compete to guide goal-directed behavior; thus, the representational system can override the habit system. While the habit system may lead an individual to be influenced by perceptual information, the representational system, or language-guided reflection, overrides perceptually driven behaviors in favor of more mature goal-directed behavior. This model serves to explain the relationship between executive functions and language throughout the lifespan.

Vocabulary. Given the HCSM's claims regarding the importance of verbal ability for executive function performance, ${ }^{18}$ it is probable that vocabulary, in particular, is important. Cascia and Barr ${ }^{60}$ reported on expressive and receptive vocabulary in relation to executive 
functions in children and adolescents with ASD, ranging in age from 9 to 18 years (mean age $=14.65$ years). They found that receptive and expressive vocabulary standard scores were related to parent and teacher ratings of executive functions. Specifically, expressive and receptive vocabulary scores were related to parent ratings of attention, emotion regulation, and inhibitory control. Based on the teacher ratings, receptive vocabulary was related to attention and organization, and both expressive and receptive vocabulary scores were related to emotion regulation, flexibility, inhibitory control, initiation, and self-monitoring. The goal of their study was to examine how executive function mediates the relationship between vocabulary and empathy; they found that both vocabulary and executive functioning abilities were important for empathy. The authors discussed how more advanced vocabulary can support social interactions. The same logic/ argument can be extended to executive functioning skills, such that better executive functioning ability can support social interactions. Therefore, not only are vocabulary and executive functions related to one another, but they both underlie important social outcomes. ${ }^{60}$

Joseph and colleagues ${ }^{41}$ studied executive function and lexical skills in 34 school-age children with ASD and 31 children without ASD who were matched on age, verbal IQ, and nonverbal IQ. Children with ASD had poorer expressive and receptive vocabulary raw and standard scores. Unlike the comparison group, vocabulary scores for the children with ASD were not correlated to measures of inhibition or planning. Moreover, language and working memory were not related in either group. The authors posited that children with ASD did not use their language to internally maintain rules or to verbally encode steps to complete the tasks. These findings may suggest that children with ASD present with a deficit in verbal mediation, or the ability to use language skills internally to facilitate accomplishing goaldirected behavior.

To examine lexical processing skills in relation to executive functions, Haebig et $\mathrm{a}^{61}$ used a lexical decision task and executive function tasks that targeted shifting and updating. Groups included 30 TD children, 27 children with ASD, and 28 children with DLD. The groups were matched on receptive vocabulary growth scores. The authors found that both shifting and updating abilities predicted accuracy and reaction time on the lexical processing task. The authors posited that children, regardless of diagnosis, process language with the aid of nonlinguistic mechanisms. Therefore, they argue that, consistent with the HCSM, there exists a relationship between language and executive functions, such that executive function supports language processing. The directionality of this relationship cannot be determined from this study, however, given its cross-sectional design. ${ }^{61}$

Syntax. The HCSM also leads to the hypothesis that better syntactic skills would benefit performance on executive function tasks. Some studies have used the Clinical Evaluation of Language Fundamentals $\left(\mathrm{CELF}^{62}\right)$ to examine expressive and receptive language ability, including syntactic ability. For example, Akbar and colleagues assessed children with ASD between the ages of 8 and 19 years ( $76 \%$ male). Children completed the CELF, Fourth Edition ${ }^{62}$ to obtain a receptive, expressive, and core language score. The Children's Communication Checklist ${ }^{63}$ was used as a parent report measure of language. The authors assessed working memory, organization, shifting, and inhibition using the Delis-Kaplan Executive Function System $\left(\mathrm{DKEFS}^{64}\right.$ ), the Developmental Neuropsychological Assessment (NEPSY-II ${ }^{65}$ ), and the Wechsler Intelligence Scales For Children $\left(\mathrm{WISC}^{66}\right)$. The CELF-4 core language score was a significant predictor of working memory. Nonverbal IQ scores predicted organization and shifting; ASD severity predicted shifting as well. There were no predictors for inhibition. The authors suggested that working memory deficits in ASD may be the result of weak language ability, as working memory is also a deficit for kids with DLD. The authors also proposed that this connection between working memory, syntax, and semantics could be due to a limitation in the ability to develop and utilize verbal mediation strategies during these executive function tasks. ${ }^{11}$

Ellis Weismer and colleagues ${ }^{67}$ also used the CELF- $4^{62}$ to specifically look at associations between receptive and expressive language scores and executive functions (i.e., shifting, 
inhibition, and updating of working memory). Their participant groups included children with TD and children with ASD, who were all between the ages of 8 and 12 years. There were several associations between receptive and expressive language skills and executive functions in children with ASD. However, when the children with ASD were split into groups with typical language and language impairment, receptive language abilities were associated with different executive function domains. In the group with language impairment, receptive language was correlated with shifting; in the group with normal language, receptive language was correlated with inhibition. The authors proposed that the association between language and executive functioning skills support the HCSM, but they caution that this finding does not answer the question of directionality. ${ }^{67}$

Durrleman and Delage ${ }^{32}$ studied 5- to 16-year-old children and adolescents with ASD (mean age $=9 ; 07$ ) and DLD (mean age $=9 ; 07)$ compared with age-matched controls and younger controls matched on expressive grammar abilities. They assessed expressive grammar and specifically examined pronoun production. They used a backward digit span task to assess working memory. When they controlled for nonverbal reasoning, they found correlations between third person pronoun production and backward digit span for both the children with ASD and the children with DLD; however, they did not look at these relationships in the children with TD. The authors suggested that the production of complex grammar recruits working memory ability in children with ASD and DLD. ${ }^{32}$

Pragmatic language. Widespread and pervasive deficits in pragmatic language skills in children and adolescents with ASD make this area of language particularly interesting and important to study. Several studies have reported connections between pragmatic language and executive functions. For instance, in addition to studying the relationship between executive functions and structural language, Akbar and colleagues used the pragmatic judgment subtest of the Comprehensive Assessment of Spoken Language (CASL ${ }^{68}$ ) to examine the relationship between executive functions and pragmatic language in their group of 8- to 19-year-old participants with ASD. They found that the pragmatic judgment score of the CASL predicted working memory in children with ASD as measured by a letter and number sequencing task, but it did not predict other aspects of executive functions, including shifting and inhibition. ${ }^{11}$ The authors proposed that syntactic skills and pragmatic skills both contribute to the ability to perform well on tasks of working memory.

Other studies have examined the expressive pragmatic skills in children and adolescents with ASD. For example, McEvoy and colleagues ${ }^{21}$ assessed preschool-age children with ASD, developmental delay, and TD. Children completed a battery of executive function tasks, and the Early Social Communication Scales ${ }^{69}$ was used to measure joint attention and social interaction. The authors found that the children with ASD had lower scores in social interaction compared with the other two groups. The children with ASD were also not able to flexibly change sets on the spatial reversal task, and they had more perseverative errors than the two other groups, meaning that the children with ASD persisted in using a previously reinforced strategy even though this strategy no longer resulted in a reward. In addition, a significant relationship was observed between social interaction behaviors and executive functioning skills across groups. The social interactions had specific goals (e.g., rolling a ball back and forth with the examiner), and the ability to maintain and achieve these goals may be reflected by the relationship between social interaction behaviors and executive function skills. Finally, there was a relationship between social interaction behaviors and joint attention, suggesting that this relationship may account for the link between social interaction and executive functions, as joint attention was also related to perseverations on the spatial reversal task. ${ }^{21}$

Gilotty and colleagues ${ }^{13}$ examined executive function skills in relation to adaptive skills in children and adolescents with ASD, ranging in age from 6 to 17 years. They found relationships between executive functioning skills and the communication and socialization domains of the Vineland Adaptive Behavior Scales. Specifically, poor communication domain scores and 
social domain scores were related to less initiation and worse working memory skills. This supports the hypothesis that working memory and aspects of language and communication are associated in this population. ${ }^{13}$ Pugliese et $\mathrm{al}^{70}$ reported similar findings, such that parent reports of executive functioning skills predicted socialization and communication scores on the Vineland Adaptive Behavior Scales $\left(\mathrm{VABS}^{71}\right)$ in school-age children with high-functioning ASD. ${ }^{70}$

In sum, language abilities (overall language and pragmatic language, specifically) appear to be particularly important for working memory in this population. ${ }^{11,13,32,67}$ It is also possible that working memory underlies performance on language-based tasks, such as a lexical decision task. ${ }^{61}$ Findings regarding the relationship between executive functions and expressive and receptive vocabulary knowledge appear to be mixed. ${ }^{41,60}$ Given these findings, and the premise of the HCSM, executive function and language certainly appear to be related; however, it is difficult to determine the directionality of the relationship between these two skills. Regardless, deficits in these domains in children with ASD warrant further research, particularly in how clinical practice can improve these two skills.

\section{IMPACT OF INTERVENTION}

The relationships between executive functions and domains of language ability (e.g., vocabulary, syntax, and pragmatics) in ASD raise questions surrounding the impact of intervention. Specifically, if structural and/or pragmatic language skills are in fact associated with executive functions, one could argue that an intervention that targets one domain would lead to generalized improvements in the other domain. The HCSM would purport that improvements in language ability would lead to improvements in executive functions, although results reported by Haebig and colleagues ${ }^{61}$ would imply the opposite, such that executive function would support language performance. In line with the HCSM, a few studies have looked at executive functions as an outcome after administering a social-based pragmatic intervention.

Stichter and colleagues examined the effectiveness of a social competence intervention for elementary school students with high-functio- ning ASD. ${ }^{72}$ They used the Social Competence Intervention-Elementary $\left(\mathrm{SCI}-\mathrm{E}^{72}\right)$ program for participants with ASD between 6 and 10 years of age (mean age $=8.77$ years). IQ scores ranged from 72 to 124 . This intervention targeted social communication areas including turn-taking, recognizing feelings and facial expressions in others, sharing ideas, and problem solving. They obtained several measurements pre- and postintervention, including the parent-report and teacher-report Social Responsiveness $\mathrm{Scale}^{73}$ as a measure of social functioning, and tasks that measured emotion recognition, executive functions, and theory of mind. Executive functions were measured through parent report and direct assessment. The intervention resulted in improvements in theory of mind, as well as better problem-solving abilities as measured through the direct assessment. Parent perceptions of social functioning and executive functions also increased. ${ }^{74}$

Morgan and colleagues conducted a cluster randomized trial to examine the effects of classroom Social, Communication, Emotional Regulation, and Transactional Support $\left(\right.$ SCERTS $\left.^{75}\right)$ intervention in comparison to a school-based education that included ASD training modules. Specifically, they were interested in executive functions and social outcomes, in addition to active engagement in the classroom and adaptive behaviors, for children with ASD. One hundred eighteen children with ASD were in the classroom SCERTS intervention group (mean age $=6.82$ years), and 79 children were assigned to the ASD training modules group (mean age $=6.77$ years). Participants in these groups were not significantly different on age, race, ethnicity, gender, ASD severity, or pretest IQ measures. They found that the group receiving the classroom SCERTS intervention had better outcomes in social skills and executive functions. One explanation for these findings is that the SCERTS model is inherently social, in that the main goal is to increase the student's engagement and social communication. However, it is important to note that the outcome measures for social skills and executive functions were teacher-report scales, which may have biased the results as the teachers knew which treatment they were administering. ${ }^{76}$ 
One study examined an executive function intervention and its impact on social skills. Kenworthy and colleagues ${ }^{77}$ conducted a randomized control trial of Unstuck and On Target (UOT), which is an executive function intervention. Forty-seven children with ASD were assigned to the UOT intervention, while 20 children with ASD were assigned to a social skills intervention. Children were between 7 and 11 years old and all had IQ scores above 70. The children who received the UOT intervention had greater improvements in domains of executive function, including flexibility and planning, compared with the children who received the control social skills intervention, although both groups demonstrated improvements on pre- and postintervention assessments. Children in both groups also made improvements in social skills, indicating that treatments targeting executive function may also help pragmatic language skills. ${ }^{77}$

\section{CLINICAL IMPLICATIONS}

Extending the complex and often contradictory findings of both empirical and clinical research to practice is difficult given the heterogeneity of ASD. This is also complicated by the differences between studies in terms of the tasks used for assessment, particularly the use of verbal- or visual-based tasks; the comparison groups selected, including children with ADHD, DLD, or TD; and the methods used for group matching. Specifically, groups matched on language do not yield differences in executive function performance between children with $\mathrm{TD}$ and $\mathrm{ASD},{ }^{61}$ but matching on IQ or age inconsistently yields differences between groups. ${ }^{42-44,46,48,53}$ Despite the variability in findings, some key patterns do emerge from these studies.

Social communication-based interventions appear to have a positive impact on executive function. Several studies that targeted social skills in elementary school children found improvements in executive functions after treatment. One theory for this is that children in social-based interventions learn to self-reflect, and they also learn to observe the behaviors of peers. If children strengthen their ability to observe and understand their own behaviors and the behaviors of their peers, it is likely that they will use these self-reflection skills during executive function tasks. If verbal mediation does play a role in executive function tasks, then perhaps this skill is enhanced during a social-based intervention, and increased practice translates to executive functions. Conversely, just as social-based interventions can result in improvements in executive functions, there is evidence that executive function-based intervention can result in improvements in social skills. $^{77}$

Structural language ability is important for performance on tasks of executive functions. Connections between structural language (lexical and syntactic skills) and executive functions support the HCSM, such that language guides reflection on behavior and ultimately impacts performance on these cognitive tasks. For instance, several studies note that working memory and syntax are related ${ }^{11,32}$ and lexical abilities appear to be important for shifting and inhibition. ${ }^{60,61}$ These relationships support the hypothesis that the ability to use linguistic skills to drive one's behavior is important for tasks of executive functions. Thus, if this hypothesis is correct, the clinical implications are that focusing on the development of vocabulary and syntax may ultimately benefit children with ASD both academically and socially. However, further research is needed to test this hypothesis.

Consideration of ASD symptom severity. ASD symptomatology and severity impacts the presence of both restricted and repetitive behaviors as well as social deficits, which are the two core deficits in ASD. Several studies have found connections between executive function and ASD symptomatology and/or severity. Thus, ASD symptomatology and/or severity is an important factor to consider in both research and clinical practice. However, it is challenging to disentangle these features, and it is not clear if increased severity impacts performance on executive function tasks, or if impairments in executive function result in presentation of more severe symptoms. For example, children with ASD tend to make more perseverative errors on tasks measuring shifting in comparison to children with DLD or ADHD, ${ }^{46,50}$ and these errors are potentially positively correlated 
with ASD severity and symptomology. A treatment approach must be carefully selected, knowing that these errors and behaviors are likely a product of this core deficit of ASD. It is challenging to draw direct conclusions from present work, however, because the majority of studies examining executive functions and language include children with high-functioning ASD.

In addition to considering ASD severity and symptomology, it is still important to comprehensively account for an individual's profile, including strengths, weaknesses, and motivators, before administering treatment. Consideration of the individual's profile, use of clinical expertise and judgment, and implementation of researchbased treatment need to be integrated to perform evidence-based practice.

\section{CONCLUSIONS}

There are large variations in findings and contradictory reports of executive function performance and its relation to language ability in children and adolescents with ASD, and future work should aim to carefully characterize the participant samples to aid in presenting clear conclusions within a heterogeneous disorder. It remains evident that language and executive function are important for several aspects of daily life, impacting outcomes such as scholastic achievement, peer victimization, and reciprocal relationships. ${ }^{14-16}$ Given that both executive functions and language ultimately have profound effects on daily life, and that one can be influenced by targeting the other in intervention, it is important that future research continues to examine the interplay between these two domains.

\section{CONFLICT OF INTEREST}

None declared.

\section{ACKNOWLEDGMENTS}

This work was supported in part by a training grant from the National Institute on Deafness and Other Communication Disorders (T32 DC05359; PI: Susan Ellis Weismer), as well as a core grant to the Waisman Center from the National Institute of Child Health and Human Development (U54 HD090256, PI: Qiang
Chang). We would also like to thank Dr. Margarita Kaushanskaya, Dr. Stacy Wagovich, and Dr. Susan Ellis Weismer for their thoughtful input on this manuscript.

\section{DISCLOSURES}

Financial: This work was supported in part by a training grant from the National Institute on Deafness and Other Communication Disorders (T32 DC05359; PI: Susan Ellis Weismer), as well as a core grant to the Waisman Center from the National Institute of Child Health and Human Development (U54 HD090256, PI: Qiang Chang).

Nonfinancial: No relevant relationships exist

\section{REFERENCES}

1. American Psychiatric Association. Diagnostic and Statistical Manual of Mental Disorders: DSM-5. 2013

2. Baio J, Wiggins L, Christensen DL, et al. Prevalence of autism spectrum disorder among children aged 8 years - Autism and Developmental Disabilities Monitoring Network, 11 sites, United States, 2014. MMWR Surveill Summ 2018;67(06):1-23

3. Pellicano E. The development of executive function in autism. Autism Res Treat 2012;2012:146132

4. Craig F, Margari F, Legrottaglie AR, Palumbi R, de Giambattista C, Margari L. A review of executive function deficits in autism spectrum disorder and attention-deficit/hyperactivity disorder. Neuropsychiatr Dis Treat 2016;12:1191-1202

5. Leung RC, Zakzanis KK. Brief report: cognitive flexibility in autism spectrum disorders: a quantitative review. J Autism Dev Disord 2014;44(10):2628-2645

6. Hill EL. Executive dysfunction in autism. Trends Cogn Sci 2004;8(01):26-32

7. Kenworthy L, Yerys BE, Anthony LG, Wallace GL. Understanding executive control in autism spectrum disorders in the lab and in the real world. Neuropsychol Rev 2008;18(04):320-338

8. Kaushanskaya M, Park JS, Gangopadhyay I, Davidson MM, Weismer SE. The relationship between executive functions and language abilities in children: a latent variables approach. J Speech Lang Hear Res 2017;60(04):912-923

9. Miyake A, Friedman NP, Emerson MJ, Witzki AH, Howerter A, Wager TD. The unity and diversity of executive functions and their contributions to complex "Frontal Lobe" tasks: a latent variable analysis. Cognit Psychol 2000;41(01):49-100

10. Zelazo PD, Craik FIM, Booth L. Executive function across the life span. Acta Psychol (Amst) 2004; 115(2-3):167-183 
11. Akbar M, Loomis R, Paul R. The interplay of language on executive functions in children with ASD. Res Autism Spectr Disord 2013;7(03): 494-501

12. Gruber O, Goschke T. Executive control emerging from dynamic interactions between brain systems mediating language, working memory and attentional processes. Acta Psychol (Amst) 2004;115(23):105-121

13. Gilotty L, Kenworthy L, Sirian L, Black DO, Wagner AE. Adaptive skills and executive function in autism spectrum disorders. Child Neuropsychol 2002;8(04):241-248

14. St Clair-Thompson HL, Gathercole SE. Executive functions and achievements in school: shifting, updating, inhibition, and working memory. QJ Exp Psychol (Hove) 2006;59(04):745-759

15. Kloosterman PH, Kelley EA, Parker JDA, Craig WM. Executive functioning as a predictor of peer victimization in adolescents with and without an autism spectrum disorder. Res Autism Spectr Disord 2014;8(03):244-254

16. Whitehouse AJO, Watt HJ, Line EA, Bishop DVM. Adult psychosocial outcomes of children with specific language impairment, pragmatic language impairment and autism. Int J Lang Commun Disord 2009;44(04):511-528

17. Long MR, Horton WS, Rohde H, Sorace A. Individual differences in switching and inhibition predict perspective-taking across the lifespan. Cognition 2018;170(April):25-30

18. Marcovitch S, Zelazo PD. A hierarchical competing systems model of the emergence and early development of executive function. Dev Sci 2009; 12(01):1-18

19. Hale CM, Tager-Flusberg H. Social communication in children with autism: the relationship between theory of mind and discourse development. Autism 2005;9(02):157-178

20. Capps L, Kehres J, Sigman M. Conversational abilities among children with Autism and children with developmental delays. Autism 1998;2(04): 325-344

21. McEvoy RE, Rogers SJ, Pennington BF. Executive function and social communication deficits in young autistic children. J Child Psychol Psychiatry 1993;34(04):563-578

22. Kissine M. Pragmatics, cognitive flexibility and autism spectrum disorders. Mind Lang 2012;27 (01):1-28

23. Eigsti I, De Marchena AB, Schuh JM, Kelley E. Language acquisition in autism spectrum disorders : a developmental review. Res Autism Spectr Disord 2011;5:681-691

24. Arnold JE, Bennetto L, Diehl JJ. Reference production in young speakers with and without autism: effects of discourse status and processing constraints. Cognition 2009;110(02):131-146
25. Adams C, Green J, Gilchrist A, Cox A. Conversational behaviour of children with Asperger syndrome and conduct disorder. J Child Psychol Psychiatry 2002;43(05):679-690

26. Kover ST, McDuffie AS, Hagerman RJ, Abbeduto L. Receptive vocabulary in boys with autism spectrum disorder: cross-sectional developmental trajectories. J Autism Dev Disord 2013;43(11): 2696-2709

27. Hudry K, Leadbitter K, Temple K, et al; PACT Consortium. Preschoolers with autism show greater impairment in receptive compared with expressive language abilities. Int J Lang Commun Disord 2010;45(06):681-690

28. Ellis Weismer S, Lord C, Esler A. Early language patterns of toddlers on the autism spectrum compared to toddlers with developmental delay. J Autism Dev Disord 2010;40(10):1259-1273

29. Jarrold C, Boucher J, Russell J. Language profiles in children with autism. Autism 1997;1:57-76

30. Volden J, Smith IM, Szatmari P, et al. Using the Preschool Language Scale, Fourth Edition to characterize language in preschoolers with autism spectrum disorders. Am J Speech-Language Pathol 2011;20:200-208

31. Hobson RP, Lee A, Hobson JA. Personal pronouns and communicative engagement in autism. J Autism Dev Disord 2010;40(06):653-664

32. Durrleman S, Delage H. Autism spectrum disorder and specific language impairment: overlaps in syntactic profiles. Lang Acquis 2016;23(04):361-386

33. De Mulder H. Developing communicative competence: a longitudinal study of the acquisition of mental state terms and indirect requests. J Child Lang 2015;42(05):969-1005

34. Pierce $S$, Bartolucci G. A syntactic investigation of verbal autistic, mentally retarded, and normal children. J Autism Child Schizophr 1977;7(02): 121-134

35. Park CJ, Yelland GW, Taffe JR, Gray KM. Morphological and syntactic skills in language samples of pre school aged children with autism: atypical development? Int J Speech-Language Pathol 2012;14(02):95-108

36. Eigsti IM, Bennetto L, Dadlani MB. Beyond pragmatics: morphosyntactic development in autism. J Autism Dev Disord 2007;37(06): 1007-1023

37. Ambridge B, Bannard C, Jackson GH. Is grammar spared in autism spectrum disorder? Data from judgments of verb argument structure overgeneralization errors. J Autism Dev Disord 2015;45(10): 3288-3296

38. Modyanova N, Perovic A, Wexler K. Grammar is differentially impaired in subgroups of autism spectrum disorders: evidence from an investigation of tense marking and morphosyntax. Front Psychol 2017;8:320 
39. Kjelgaard MM, Tager-Flusberg H. An investigation of language impairment in autism: implications for genetic subgroups. Lang Cogn Process 2001;16(2-3):287-308

40. Roberts JA, Rice ML, Tager-Flusberg H. Tense marking in children with autism. Appl Psycholinguist 2004;25(03):429-448

41. Joseph RM, McGrath LM, Tager-Flusberg H. Executive dysfunction and its relation to language ability in verbal school-age children with autism. Dev Neuropsychol 2005;27(03):361-378

42. Corbett BA, Constantine LJ, Hendren R, Rocke $\mathrm{D}$, Ozonoff S. Examining executive functioning in children with autism spectrum disorder, attention deficit hyperactivity disorder and typical development. Psychiatry Res 2009;166(2-3):210-222

43. Kado Y, Sanada S, Yanagihara M, et al. Executive function in children with pervasive developmental disorder and attention-deficit/hyperactivity disorder assessed by the Keio version of the Wisconsin card sorting test. Brain Dev 2012;34(05):354-359

44. Yang J, Zhou S, Yao S, Su L, McWhinnie C. The relationship between theory of mind and executive function in a sample of children from mainland China. Child Psychiatry Hum Dev 2009;40(02): 169-182

45. Pellicano E. Individual differences in executive function and central coherence predict developmental changes in theory of mind in autism. Dev Psychol 2010;46(02):530-544

46. Liss M, Fein D, Allen D, et al. Executive functioning in high-functioning children with autism. J Child Psychol Psychiatry 2001;42(02):261-270

47. Kimhi Y, Shoam-Kugelmas D, Agam Ben-Artzi G, Ben-Moshe I, Bauminger-Zviely N. Theory of mind and executive function in preschoolers with typical development versus intellectually able preschoolers with autism spectrum disorder. J Autism Dev Disord 2014;44(09):2341-2354

48. Roelofs RL, Visser EM, Berger HJC, Prins JB, Van Schrojenstein Lantman-De Valk HMJ, Teunisse JP. Executive functioning in individuals with intellectual disabilities and autism spectrum disorders. J Intellect Disabil Res 2015;59(02):125-137

49. Heaton R, Chelune G, Talley J, Kay G, Curtis G. Wisconsin Card Sorting Test (WCST) Manual Revised and Expanded. Odessa, FL: Psychological Assessment Resources; 1993

50. Ozonoff S, Jensen J. Brief report: specific executive function profiles in three neurodevelopmental disorders. J Autism Dev Disord 1999;29(02): 171-177

51. Gioia GA, Isquith PK, Guy SC, Kenworthy L. Behavior Rating of Executive Function. Lutz, FL: Psychological Assessment Resources; 2000

52. Roth RM, Isquith PK, Gioia GA. Behavior Rating Inventory of Executive Function - Adult Version.
Lutz, FL: Psychological Assessment Resources; 2005

53. Durrleman S, Franck J. Exploring links between language and cognition in autism spectrum disorders: complement sentences, false belief, and executive functioning. J Commun Disord 2015;54:15-31

54. Golden CJ. Stroop Color and Word Test: A Manual for Clinical and Experimental Uses. 1978

55. Johnston K, Madden AK, Bramham J, Russell AJ. Response inhibition in adults with autism spectrum disorder compared to attention deficit/hyperactivity disorder. J Autism Dev Disord 2011;41(07):903-912

56. Burgess PW, Shallice T. The Hayling and Brixton Tests. Bury St Edmonds: Thames Valley Test Company; 1997

57. Kashima H, Kato M. Wisconsin Card Sorting Test (Keio Version) (KWCST) [in Japanese]. Brain Sci Ment Disord 1995;6:209-2016

58. Hill AP, van Santen J, Gorman K, Langhorst BH, Fombonne E. Memory in language-impaired children with and without autism. J Neurodev Disord 2015;7(01):19

59. Ozonoff S, McEvoy RE. A longitudinal study of executive function and theory of mind development in autism. Dev Psychopathol 1994;6(03):415-431

60. Cascia J, Barr JJ. Associations among vocabulary, executive function skills and empathy in individuals with autism spectrum disorder. J Appl Res Intellect Disabil 2017;30(04):627-637

61. Haebig E, Kaushanskaya M, Ellis Weismer S. Lexical processing in school-age children with autism spectrum disorder and children with specific language impairment: the role of semantics. J Autism Dev Disord 2015;45(12):4109-4123

62. Semel E, Wiig EH, Secord WA. Clinical Evaluation of Language Fundamentals - 4. San Antonio, TX: The Psychological Corporation; 2003

63. Bishop DVM. The Children's Communication Checklist Second Edition (CCC-2). London: The Psychological Corporation; 2003

64. Delis DC, Kaplan E, Kramer JH. The DelisKaplan Executive Function System: Examiner's Manual. San Antonio, TX: The Psychological Corporation; 2001

65. Korkman M, Kirk U, Kemp SL. NEPSY-II. San Antonio, TX: The Psychological Corporation; 2007

66. Wechsler D. Wechsler Abbreviated Scale of Intelligence - Second Edition. San Antonio, TX: NCS Pearson; 2011

67. Ellis Weismer S, Kaushanskaya M, Larson C, Mathée J, Bolt D. Executive function skills in school-age children with autism spectrum disorder: association with language abilities. J Speech Lang Hear Res 2018;61(11):2641-2658

68. Carrow-Woolfolk E. Comprehensive Assessment of Spoken Language (CASL). Circle Pines, MN: American Guidance Service; 1999 
69. Seibert JM, Hogan AE, Mundy PC. Assessing social communication skills in infancy. Top Early Child Spec Educ 1987;7:32-48

70. Pugliese CE, Anthony L, Strang JF, Dudley K, Wallace GL, Kenworthy L. Increasing adaptive behavior skill deficits from childhood to adolescence in autism spectrum disorder: role of executive function. J Autism Dev Disord 2015;45(06): 1579-1587

71. Sparrow SS, Cicchetti DV, Balla DA. Vineland Adaptive Behavior Scales: Survey Form (2nd ed.). Circle Pines, MN: American Guidance Service; 2005

72. Stichter JP, Herzog MJ, Visovsky K, et al. Social competence intervention for youth with Asperger Syndrome and high-functioning autism: an initial investigation. J Autism Dev Disord 2010;40(09): 1067-1079

73. Constantino JN, Gruber C. The Social Responsiveness Scale. Los Angeles, CA: Western Psychological Services; 2005
74. Stichter JP, O'Connor KV, Herzog MJ, Lierheimer K, McGhee SD. Social competence intervention for elementary students with Asperger's syndrome and high functioning autism. J Autism Dev Disord 2012;42(03):354-366

75. Prizant BM, Wetherby AM, Rubin E, Laurent AC, Rydell JP. The SCERTS model: Volume I Assessment; Volume II Program Planning and Intervention. Baltimore, MD: Brookes Publishing; 2006

76. Morgan L, Hooker JL, Sparapani N, Reinhardt VP, Schatschneider C, Wetherby AM. Cluster randomized trial of the classroom SCERTS intervention for elementary students with autism spectrum disorder. J Consult Clin Psychol 2018;86(07): 631-644

77. Kenworthy L, Anthony LG, Naiman $\mathrm{DQ}_{2}$ et al. Randomized controlled effectiveness trial of executive function intervention for children on the autism spectrum. J Child Psychol Psychiatry 2014;55(04):374-383 